Artículo

\title{
Análisis de adaptabilidad de Leucaena collinsii en la microcuenca Tula, México
}

\author{
Julio Cesar Buendía-Espinoza ${ }^{1}$ \\ Irma Díaz-Aguilar ${ }^{\S}$ \\ Jesús Eduardo Cahuich-Damián ${ }^{1}$ \\ Arturo Morales-García ${ }^{3}$ \\ Pedro Arturo Martínez-Hernández ${ }^{4}$ \\ Mayra Clementina Zamora-Elizalde ${ }^{1}$
}

${ }^{1}$ Maestría en Agroforestería para el Desarrollo Sostenible-Departamento de Suelos-Universidad Autónoma Chapingo. Carretera México-Texcoco km 38.5, Texcoco, Estado de México. CP. 56230. (damian.326@hotmail.com; zamora.elizalde.231195@gmail.com). ${ }^{2}$ Posgrado en Edafología-Colegio de Postgraduados. Carretera México-Texcoco km 36.5, Texcoco, Estado de México. CP. 56230. (idiaz@ualberta.ca). ${ }^{3}$ Ingeniería en Recursos Naturales Renovables-Departamento de Suelos-Universidad Autónoma Chapingo. (arturomgarcia09@gmail.com). ${ }^{4}$ Posgrado en Producción Animal-Departamento de Zootecnia-Universidad Autónoma Chapingo. (arturomrtz@ hotmail.com).

Autor para correspondencia: idiaz@colpos.mx

\section{Resumen}

En las últimas décadas, el deterioro de los recursos naturales en México se ha incrementado debido a los cambios del uso del suelo y a la urbanización creciente en áreas de alta vulnerabilidad ecológica y bajos niveles de productividad. La microcuenca Tula, México está ubicada en una zona de clima semiseco donde la degradación del suelo y el incremento de la población están ejerciendo un fuerte impacto en la conservación del suelo. El objetivo de este estudio fue determinar las zonas aptas para el desarrollo óptimo de la especie Leucaena collinsii Britton \& Rose en la microcuenca Tula, a través del modelo de nicho ecológico de máxima entropía, MaxEnt, con fines de rehabilitación del suelo. Para el modelado, se utilizaron 19 variables ambientales y 8 registros de presencia, obtenidas de las bases de datos GBIF y MEXU. Los resultados de la modelación indicaron que las variables con mayor contribución fueron la temperatura y la evapotranspiración, y el modelo tiene buena capacidad de predicción ( $p$-valor $=0.013)$, de acuerdo con la prueba de significancia con $\alpha=0.05$, basada en la técnica de Jackniffie. En el modelo, la distribución potencial de la especie fue baja, con apenas $8 \%$ de la superficie de la microcuenca, pero se registró una alta sobrevivencia (100\%), en la validación experimental. Se concluyó que existen escasas zonas de adaptabilidad para la especie, en la microcuenca Tula; sin embargo, su alta sobrevivencia en la parcela experimental determinó que su introducción es una opción para la rehabilitación del suelo.

Palabras clave: distribución potencial, degradación del suelo, modelado de nicho ecológico, sobrevivencia, rehabilitación.

Recibido: junio de 2020

Aceptado: agosto de 2020 


\section{Introducción}

En las últimas décadas, el deterioro de los recursos naturales en México ha ido incrementando, el cual en muchos casos es irreversible. Los actuales ritmos del cambio del uso del suelo han subestimado los daños que las tecnologías y actividades agropecuarias y forestales producen en los recursos naturales. Aunado a lo anterior, el proceso de urbanización como tendencia creciente en áreas de alta vulnerabilidad ecológica y bajos niveles de productividad, como son las zonas de climas seco y semiseco, y suelos someros en lomeríos y con pendientes pronunciadas.

La microcuenca Tula, México está ubicada en una zona donde convergen dos diferentes tipos de climas: templado subhúmedos y semisecos (INEGI, 2017a). Sin embargo, en el área de clima semiseco es donde la degradación del suelo es una característica común y donde la población de los 14 municipios que la conforman se ha incrementado en un promedio de $2 \%$ anual, alcanzando un total de 288196 habitantes en al año 2010 (INEGI, 2013a).

Este incremento en la población ha causado una mayor presión sobre los recursos naturales de la región, además de problemas asociados al cambio de uso del suelo (Zamora et al., 2020). También, las áreas de extracción de materiales para construcción, las superficies agrícolas y la mancha urbana han aumentado (INEGI, 2017a, 2017b, 2017c), provocando una mayor degradación del suelo debido a la perdida de la cobertura vegetal.

Por consiguiente, surge la necesidad de buscar alternativas de rehabilitación, recuperación y conservación de suelos en las áreas que han perdido su productividad, además de prácticas agronómicas o vegetativas (Zavala-Cruz et al., 2011). Una alternativa es la introducción de plantas de uso múltiple (producción forrajera, cubierta vegetal y conservación del suelo), que tengan un óptimo desarrollo en la región.

La especie Leucaena collinsii Britton \& Rose se distribuye del sur de México hasta Guatemala y pertenece a la familia Fabaceae (Zárate-Pedroche, 1998). En México, ésta es utilizada como forraje (por su bajo contenido de taninos y mimosina y alta capacidad de rebrote), en reforestaciones para el control de erosión, como árbol de sombra y fijadora de nitrógeno (Zárate-Pedroche, 1998; Mullen et al., 2003; Wencomo y Ortiz, 2010; Pinto et al., 2013).

Entre sus características principales destacan su notable tolerancia al estrés hídrico debido a su sistema radical pivotante que le permite alcanzar la humedad en las capas profundas del suelo, resistencia al psílido (insecto fitófago, Heteropsylla cubana) que son una de las plagas principales de su género y su adaptación en una amplia variedad de suelos, desde neutros hasta alcalinos (Hernández et al., 1987; Zárate-Pedroche, 1998; Martínez-Hernández et al., 2019).

Sin embargo, hasta la fecha, no ha habido intentos por determinar su distribución geográfica potencial a escalas finas, a pesar de que es una especie de distribución cosmopolita y que se pueden introducir en áreas con precipitaciones escasas. Por tanto, conocer la distribución de esta especie ayudaría a determinar las áreas en las que es conveniente introducirla con fines de rehabilitación del suelo. 
Por otra parte, la distribución de especies se rige por varios factores entre los más importantes son los ambientales, ya que su sobrevivencia y desarrollo está determinado por un rango de condiciones ambientales, que son los indicadores para determinar su respectivo nicho ecológico entre otros factores (López, 2007; Peterson et al., 2008; Soberón y Nakamura, 2009).

En los últimos años se han desarrollado más de 15 algoritmos para modelar nichos ecológicos de especies y poder obtener su distribución potencial (Elith et al., 2006), estos algoritmos se basan en el concepto de nicho ecológico establecido por Hutchinson (1957), quien relaciona la información biológica con la información ambiental, para determinar áreas en donde no existen registros de presencia de una especie pero que cumplen con las condiciones adecuadas para la sobrevivencia y desarrollo de la especie (Soberón y Peterson, 2005; López, 2007; Elith et al., 2010).

MaxEnt es un algoritmo para hacer predicciones de distribuciones geográficas de especies (Phillips et al., 2006) y se considera uno de los mejores algoritmos para modelar nichos ecológicos (Contreras-Medina et al., 2010; Elith et al., 2010), por determinar la relación entre las variables ambientales y los registros de presencia de una especie (Broennimann et al., 2007; Palma-Ordaz y Delgadillo-Rodríguez, 2014).

Es un método de inteligencia artificial que estima la probabilidad de ocurrencia de la especie buscando la distribución de máxima entropía (lo más uniforme posible) sujeta a la condición de que el valor esperado de cada variable ambiental según esta distribución coincide con su media empírica (López, 2007; Flores-Maldonado, 2014).

El resultado del modelo expresa el valor de idoneidad del hábitat para la especie como una función de las variables ambientales (Phillips et al., 2006). Un valor alto de la función de distribución en una celda determinada indica que ésta presenta condiciones muy favorables para la presencia de la especie (López, 2007; Flores-Maldonado, 2014).

La modelación de nichos ecológicos con MaxEnt ha sido empleada en varios estudios entre los que se encuentran, el de Peralta et al. (2013) con Quercus rotundifolia; Cruz-Cárdenas et al. (2014) con Liquidambar styraciflua L., Quercus rubramenta Trel. y Roldana robinsoniana (Greenm.) H. Rob. \& Brettell.; Quesada-Quirós et al. (2016) con Comarostaphylis arbutoides (Lindl.), Ilex pallida (Standl.), Myrrhidendron donnellsmithii (J. M. Coult. \& Rose), Quercus costaricensis (Liebm.) y Vaccinium consanguineum (Klotzsch) y Reynoso et al. (2018) con Pinus pseudostrobus Lindl. y Pinus oocarpa Schiede ex Schltdl.

De los anteriores estudios se demostró que el modelado de nichos ecológicos facilitó el entendimiento de los patrones potenciales de distribución de una especie y las zonas de mayor idoneidad ambiental para su establecimiento y desarrollo. Por lo anterior, el presente trabajo tiene como objetivo determinar las zonas aptas de distribución potencial para el desarrollo óptimo de la especie Leucaena collinsii Britton \& Rose en la microcuenca Tula; a través, del modelo de nicho ecológico por máxima entropía, MaxEnt, con fines de rehabilitación del suelo. 


\section{Materiales y métodos}

\section{Localización del área de estudio}

La microcuenca Tula se ubica en las coordenadas geográficas $19^{\circ} 55^{\prime} 31.05^{\prime}$ ' y $19^{\circ} 34^{\prime} 28.74$ ', latitud norte y $98^{\circ} 39^{\prime}$ '59' ' y $98^{\circ} 28^{\prime} 16.39^{\prime}$ ' latitud oeste, con una superficie de $1037.66 \mathrm{~km}^{2}$ (103 766 hectáreas) y abarca territorio tres estados de la República Mexicana: Estado de México, Hidalgo y Tlaxcala. Se encuentra inmersa en la región hidrológica 26.

Cuenca del río Panuco y pertenece a la provincia fisiográfica del eje Neovolcánico, con un rango altitudinal que varía entre 2333 a $3223 \mathrm{~m}$. Los tipos de clima son templado subhúmedo con lluvias en verano y semiseco templado, la precipitación media anual es de $600 \mathrm{~mm}$, con una temperatura media anual de $14^{\circ} \mathrm{C}$ y el suelo que predomina es el feozem háplico abarcando $65 \%$ y litosol con 20\% de la superficie en la microcuenca (INEGI, 2017a, 2017b, 2017c). Presenta diversos tipos de vegetación: bosque de táscate, bosque de pino, bosque de encino y matorral crasicaule (INEGI, 2017d).

\section{Datos de presencia}

Los registros de presencia de la especie L. collinsii, obtenidos de la base de datos de Infraestructura Mundial de Información en Biodiversidad (GBIF) y del Herbario Nacional de México (MEXU), se depuraron con la finalidad de eliminar aquellos registros ubicados fuera de los límites del territorio nacional, datos duplicados y aquellos que presentaron inconsistencias, o en su caso, sin coordenadas geográficas como lo indica Martínez-Méndez et al. (2016).

\section{Variables predictivas ambientales}

Se recopilaron y procesaron los datos de 19 variables climáticas, estacionales, propiedades del suelo, atributos topográficos, e índices normalizados de vegetación, para la modelación, manteniendo un control de calidad como lo indica Hijmans et al. (2005).

Se generaron 5 variables climáticas (BIO1, BIO3, BIO4, BIO12 Y BIO15, ver Cuadro 1) obtenidas de datos de la plataforma WorlClim Versión 1.4 (http://www.worldclim.org/) y derivadas de los valores mensuales de temperatura máxima, temperatura mínima y precipitación, que representan las condiciones de un periodo de tiempo de 1959 a 2000.

Cuadro 1. Variables predictivas ambientales utilizadas en MaxEnt para generar el modelo de nicho ecológico de Leucaena collinsii en la microcuenca Tula, México.

A) variables climáticas

$\begin{aligned} \mathrm{BIO} 1 & =\text { Temperatura media anual } \\ \mathrm{BIO} 3 & =\text { Isotermalidad }(\mathrm{BIO} 1 / \mathrm{BIO} 7) * 100 \\ \mathrm{BIO} 4 & =\text { Temperatura estacional (desviación estándar } * 100) \\ \mathrm{BIO} 12 & =\text { Precipitación anual } \\ \mathrm{BIO} 15 & =\text { Precipitación estacional }\end{aligned}$




\begin{aligned} & \multicolumn{1}{c}{ B) variables estacionales } \\ ETRA & $=$ Evapotranspiración real anual \\ ETRH & $=$ Evapotranspiración real meses húmedos \\ ETRS & $=$ Evapotranspiración real meses secos \\ PPH & $=$ Precipitación meses húmedos \\ PPS & $=$ Precipitación meses secos \\ TH & $=$ Temperatura meses húmedos \\ TS & $=$ Temperatura meses secos \\ CE & $=$ Conductividad eléctrica \\ MO & $=$ Materia orgánica \\ pH & $=$ Potencial hidrógeno \\ ASP & $=$ Orientación del terreno \\ ELEV & $=$ Elevación \\ INV & $=$ Índice normalizado de vegetación mes húmedo \\ INV & $=$ Índice normalizado de vegetación mes seco \end{aligned}

La evapotranspiración real anual (ETRA) se calculó con base en el modelo de Turc (1954): ETRA= P / [0.9 + (P/L)2] 1/2. Donde: $\mathrm{P}=$ precipitación total anual $(\mathrm{mm}) ; \mathrm{L}=300+25 \mathrm{~T}+0.05 \mathrm{~T} 3 ; \mathrm{T}=$ temperatura media anual $\left({ }^{\circ} \mathrm{C}\right)$.

Además, se generaron siete variables estacionales de acuerdo con Sánchez y Carvacho (2011); a partir, de las cinco variables anteriores con el modelo de Turc (1954), estimándose variables de evapotranspiración (ETRA, ETRH, ETRS), precipitación (PH, PS) y temperatura (TH, TS), correspondientes a los meses húmedos (mayo-octubre) y secos (noviembre-abril) (Cuadro 1).

Se incluyeron tres variables del suelo ( $\mathrm{MO}, \mathrm{CE}$ y $\mathrm{pH})$, de acuerdo a las recomendaciones de CruzCárdenas et al. (2014), generadas a partir del conjunto de datos de perfiles de suelo 1:250000 Serie II (INEGI, 2013b) y dos atributos topográficos: orientación (ASP) y altitud (ELEV), generados a partir del modelo digital de elevación, con un tamaño de pixel de $120 \mathrm{~m}$, el cual se encuentra en las bases de INEGI dentro del continuo de elevaciones mexicano 3.0 (INEGI, 2013c).

Por último, se calcularon dos índices normalizados de vegetación generados a partir de imágenes de satélite MODIS TERRA de la NASA (Didan, 2015), en periodos de los meses húmedos (INV agosto 2019) y en los meses secos (INV Enero 2019) (Cuadro 1). Todas las capas fueron homogenizadas a un tamaño de pixel de $1 \mathrm{~km}^{2}$ (0.54 arco minutos).

\section{Modelado de nichos ecológicos}

La modelación de la distribución potencial de la especie L. collinsii se realizó con el programa Maxent ver. 3.4.0, que utiliza la técnica de aprendizaje automático para seleccionar el modelo más consistente a partir la información de las variables disponibles (Phillips et al., 2006; Phillips y Dudik, 2008), además, de generar buenas predicciones aun cuando se utilizen muestras pequeñas, menores de 10 datos de presencia (Phillips et al., 2006; Pearson et al., 2007). 
Las 19 capas de las variables edafo-climáticas y los registros de presencia de la especie fueron utilizados para generar su modelo de nicho ecológico potencial. En la modelación, la configuración utilizada en el algoritmo MaxEnt fue la característica lineal-cuadrática, con un multiplicador de regularización igual a 1, para los modelos $(n=8)$, ya que registros de presencia reducidos no permiten evaluar combinaciones de complejidad, por lo que se optó por un enfoque conservador.

El formato de salida elegido para los modelos fue 'raw', que posteriormente se configuró en el algoritmo MaxEnt donde todos los modelos tuvieran el formato de salida del tipo logística, debido a la facilidad de interpretación en el sistema de información geográfica (Phillips y Dudík, 2008).

\section{Validación del modelo}

De acuerdo con Guisan y Zimmermann (2000), el rendimiento de un modelo se evalúa con un conjunto de datos de presencia independiente del que se ha usado, para calibrar el modelo. Sin embargo, esto es complicado de hacer mediante el Área Bajo la Curva, AUC, cuando se tienen datos de presencia limitados, menores a 20 , porque se tendrían que utilizar el mayor número de estos en su calibración.

En consecuencia, se realizó una prueba de significancia, basada en la técnica de Jackknife, para evaluar el rendimiento predictivo de un modelo con menos de 20 datos de presencia a través de verificar si los modelos propuestos son mejores a los realizados aleatoriamente. El método consistió en construir $n$ modelos separados con los $n$ registros de presencia depurados $(n=8)$, cada uno con $\mathrm{n}-1$ individuos $(\mathrm{n}=8-1=7)$ y evaluar la capacidad de cada uno de ellos para predecir el dato excluido del conjunto de datos.

Con el registro que se excluyó para cada modelo, se verificó el éxito o fracaso de la predicción, si coincidía en el área de distribución tomaba valor de 1 (éxito) en caso contrario 0 (fracaso). Los valores de éxito o fracaso para cada modelo, complementado con su proporción de área predicha fueron ingresados a la prueba de significancia p-value (Pearson et al., 2007), a un nivel de significancia de 0.05 . El proceso fue realizado en el ejecutable $p$-value Pearson en el software $\mathrm{R}$ (R Development Core Team, 2012).

Respecto a la evaluación de las variables ambientales, ésta se realizó con la prueba de Jackknife, con el propósito de evaluar de forma individual el aporte de cada una de ellas en el modelo e identificar la variable con información más útil por si misma (Phillips y Dudík, 2008).

\section{Evaluación de la sobrevivencia de la especie en campo}

La evaluación de sobrevivencia permite obtener una medida cuantitativa del éxito de la plantación bajo la influencia del ambiente del sitio (Ramírez, 2011) y se define como el cociente, expresado en porcentaje, entre el número de plantas que sobreviven al finalizar el control de seguimiento, y el número de plantas emergidas, expresada en porcentaje (Ramos-Font et al., 2015).

Durante los años 2018 y 2019, se estimó la sobrevivencia de la especie, en un área con alto grado de degradación del suelo, en una parcela experimental de $1500 \mathrm{~m}^{2}$ (30 x 50 m de ancho y largo, respectivamente). La parcela experimental estaba ubicada dentro de la microcuenca Tula (Figura 
1) entre las coordenadas geográficas $19^{\circ} 50^{\prime} 24$ y $19^{\circ} 49^{\prime}$ 00' de latitud norte y $98^{\circ} 42^{\prime}$ '00' de longitud oeste, a una altitud de 2473 msnm, en el Ejido de San Felipe Teotitlán, en el municipio de Nopaltepec, limitando al sur con el municipio de Axapusco, en el Estado de México.

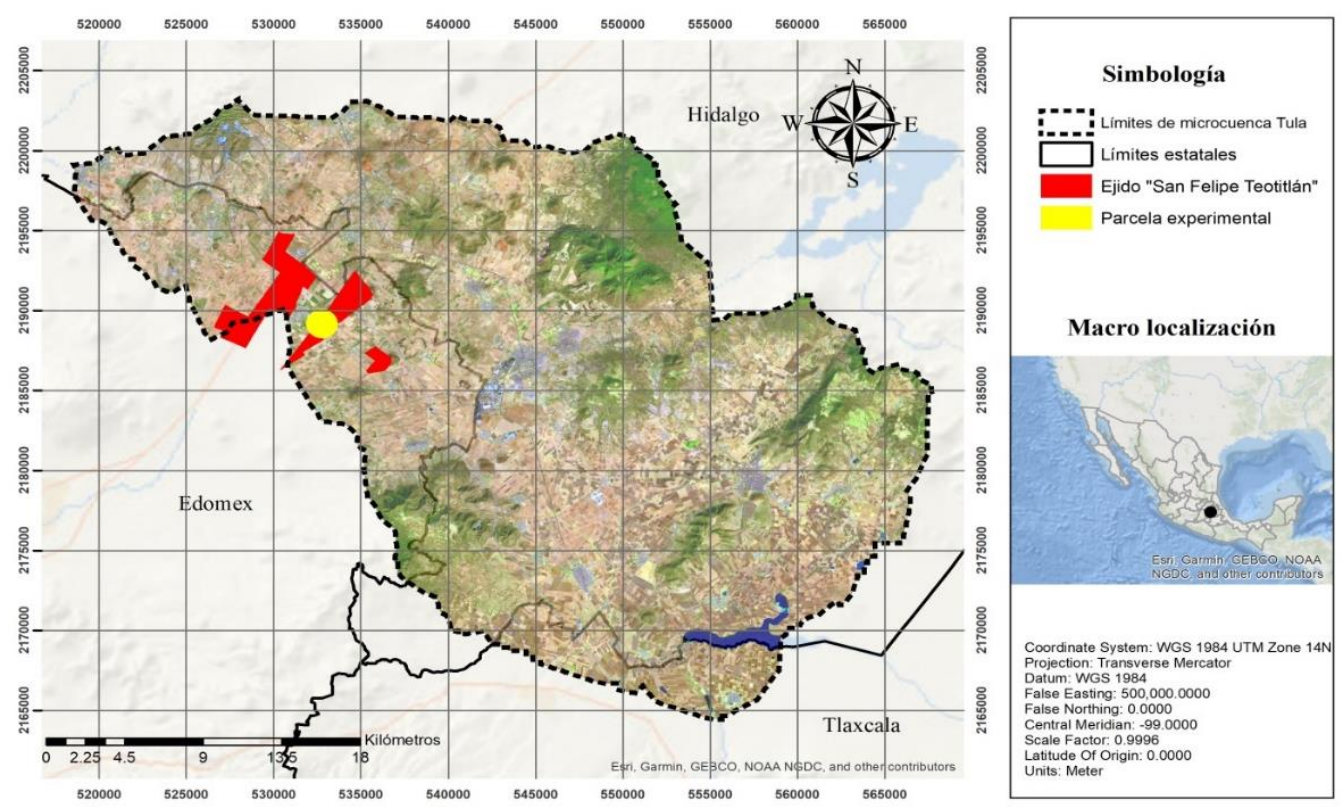

Figura 1. Localización del área experimental.

Durante el mes de julio de 2018, se trasplantaron 25 plántulas de L. collinsii, de tres meses de edad, distribuidas en forma aleatoria en la parcela experimental, a una distancia de 6 metros entre líneas y dos metros entre plantas. La estimación de la sobrevivencia se realizó en la etapa de establecimiento (tres meses después del trasplante), en octubre de 2018 y al año después del trasplante, en octubre de 2019.

\section{Resultados y discusión}

El número final de datos de presencia de L. collinsii, obtenidos de la base de datos del MEXU y GBIF, mediante el software QGIS 2.14.3, fue de 8, después del proceso de depuración de 84 registros, pertenecientes al estado de Chiapas. Los requerimientos ambientales de dicha especie se encuentran dentro de los rangos de las variables ambientales de la microcuenca Tula (ZáratePedroche, 1998; Pinto et al., 2013). Respecto a la configuración, elegida en el algoritmo MaxEnt, ésta fue con la característica lineal-cuadrática y el multiplicador de regularización igual 1, para los $\mathrm{n}=8$ modelos.

Debido a que registros reducidos no permiten evaluar combinaciones de complejidad, se optó por un enfoque conservador que permitió un mejor desempeño en el modelado de la especie. El formato de salida elegido para los modelos fue 'raw'. Los $n=8$ modelos generados se les aplicó un umbral basado en el método del valor más bajo predicho" de idoneidad de hábitat en base a los registros de entrenamiento, generando modelos binarios con valor de 1 para las zonas de distribución potencial. 
El valor de umbral fue de 0.13 , el cual se utilizó para generar el mapa de distribución potencial de L. collinsii en el software ArcMap 10.5, como resultado se obtuvo un mapa de tipo booleano. En relación con la evaluación del rendimiento del modelo, el resultado de la prueba de significancia, basada en la técnica de Jackknife, mostró que existen diferencias estadísticas significativas entre los dos tipos de modelos indicadas por el $p$-valor $=0.013$, con un nivel de significancia de 0.05 .

Esto significa que los modelos propuestos fueron mejores que los modelos realizados aleatoriamente; es decir, que los primeros tienen un buen rendimiento o capacidad de predicción y pueden servir de base como una primera aproximación para identificar zonas potenciales de establecimiento de L. collinsii (Pearson et al., 2007).

El modelo final obtenido fue con los 8 registros como entrenamiento y la característica LinealCuadrática con multiplicador de regularización igual a 1, con formato de salida del tipo logística, debido a la facilidad de interpretación en el sistema de información geográfica (Phillips y Dudík, 2008). La prueba de Jackknife indicó que las variables ambientales de mayor contribución al modelo fueron BIO 4 (Temperatura estacional, ${ }^{\circ} \mathrm{C}$ ), con $36 \%$ y la ETRAH (Evapotranspiración real anual de los meses húmedos, $\mathrm{mm} \mathrm{año}^{-1}$ ), con $31 \%$ (Figura 2).

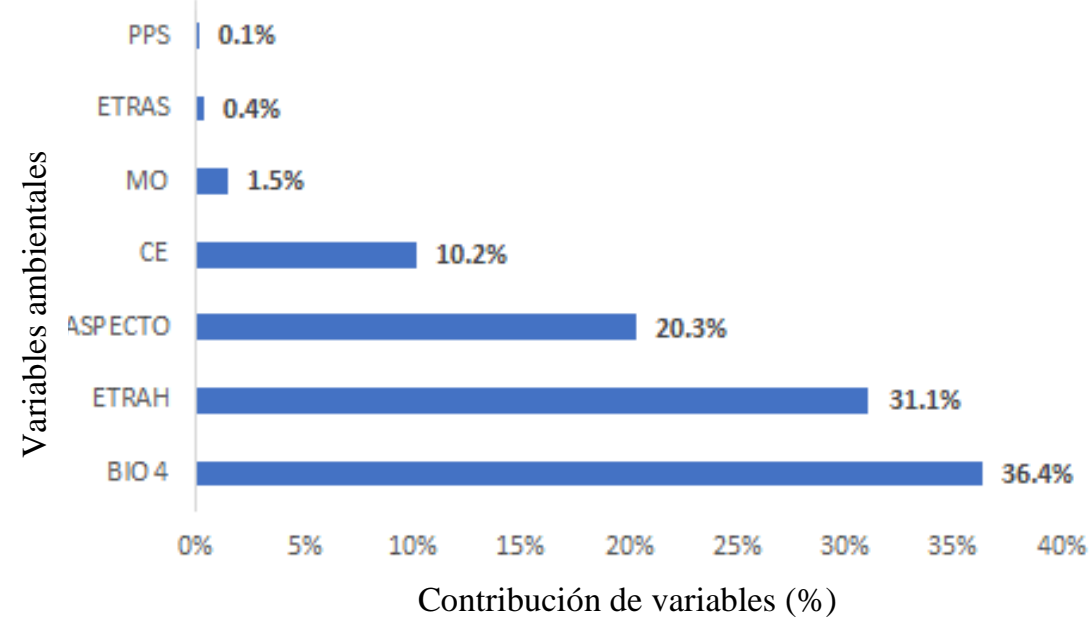

Figura 2. Contribución porcentual de las variables ambientales al modelo de $L$. collinsii.

Mostrando la importancia de la precipitación de los meses húmedos (mayo-octubre) y de la temperatura estacional en la sobrevivencia y desarrollo de la especie (Wencomo y Ortiz, 2010; Pinto et al., 2013; Martínez-Hernández et al., 2019).

Posiblemente, en sitios donde no haya una escasa profundidad del suelo (variable que no fue evaluada) sea favorable para su desarrollo en la microcuenca Tula. Al respecto, Hernández et al. (1987) mencionan que L. collinsii tiene alta capacidad de rebrote, después de las sequías, debido a su sistema radical pivotante que puede alcanzar la humedad en las capas profundas del suelo.

De la modelación del nicho ecológico de L. collinsii, se obtuvo que por las características ambientales de la microcuenca, la superficie de presencia, que es la superficie en donde es posible que se desarrolle la especie, fue de $81.9755 \mathrm{~km}^{2}$ (8 $197.55 \mathrm{ha}$ ), equivalente a $8 \%$ de la superficie de la superficie total, indicando una baja distribución respecto a la superficie de ausencia de $955.6844 \mathrm{~km}^{2}$ (95 $568.44 \mathrm{ha}$ ), equivalente a 92\% de la superficie total (Figura 3). 


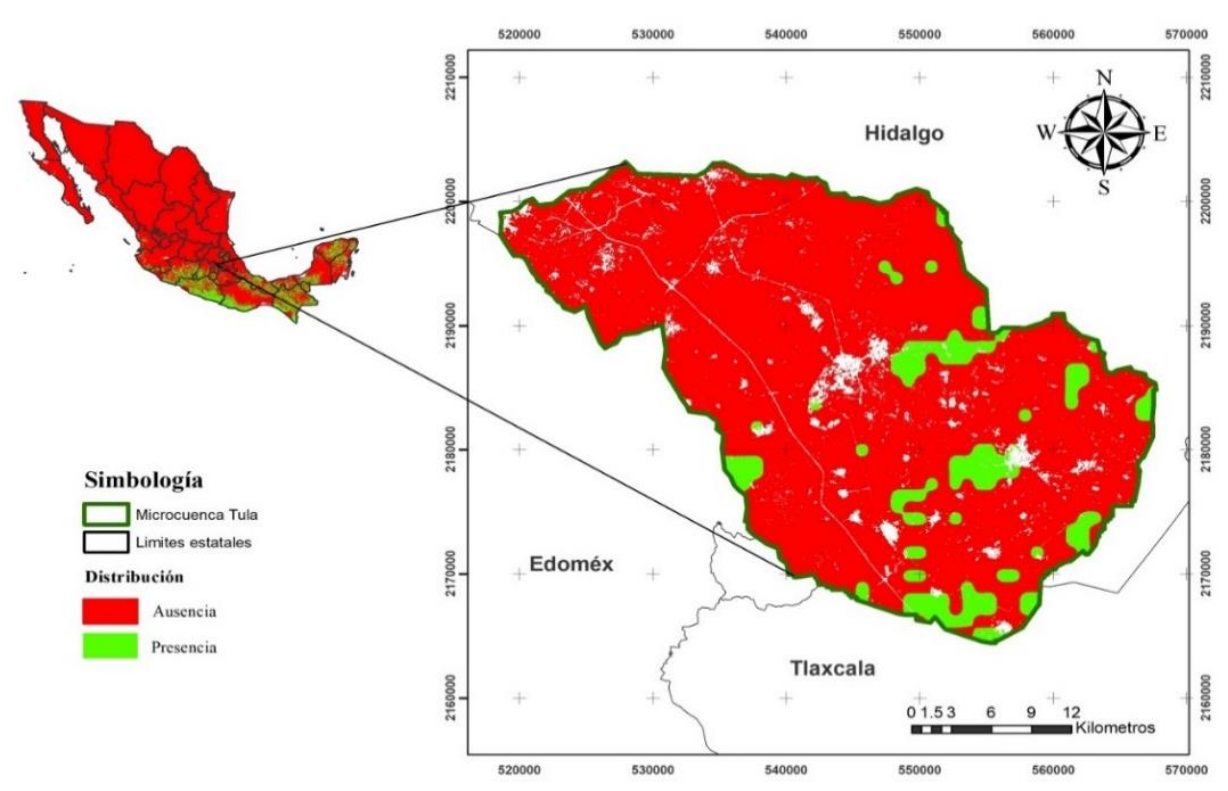

Figura 3. Distribución potencial de $L$. collinsii en la microcuenca tula, México.

Las regiones con mejores características bioclimáticas y ambientales para el favorecimiento de su adaptabilidad fueron los municipios de Apan, Almoloya, Emiliano Zapata, Tepeapulco y Singuilucan en el estado de Hidalgo, Benito Juárez, Calpulalpan y Sanctórum de Lázaro Cárdenas del estado de Tlaxcala y el municipio de Axapusco (cerca del lugar donde se llevó a cabo la validación en campo), en el Estado de México, ya que pertenecen a zonas de transición climática del régimen semiseco, al templado subhúmedo, donde se presentan condiciones de precipitación y temperatura posiblemente más idóneas para el establecimiento de L. collinsii.

Respecto a la evaluación de la sobrevivencia de la especie en campo, la cual permite medir cuantitativamente el éxito de la plantación bajo la influencia de los factores del sitio (Ramírez, 2011), ésta fue de 100\% (25 individuos), al año de trasplantada (octubre 2019). Esto coincide con lo reportado para otras especies de su género, como lo es L. leucocephala, la cual se ha estudiado en el valle del altiplano semiárido del centro-norte de México como parte de un sistema agroforestal formando barreras de muro vivo con nopal (Osuna-Ceja et al., 2019).

La alta sobrevivencia de L. collinsii se debió que tuvo una sobrevivencia alta (100\%) en la etapa de establecimiento, prácticas de manejo y buena distribución del temporal en esos años. Sin embargo, en la época de frío sufrió daño, pero debido a su alta capacidad de rebrote (Hernández et $a l .$, 1987), se recuperó. Esto significa que es una alternativa de gran interés para ser introducida con fines de rehabilitación en la microcuenca Tula, debido que puede incrementar la fertilidad del suelo (Crespo, 2008), debido a su capacidad de fijar nitrógeno atmosférico a través de la simbiosis con Rhizobium y a su capacidad de favorecer la actividad microbiana (Valpassos et al., 2007).

Pinto et al. (2013) mencionan que L. collinsii es una especie con potencial para ser utilizada como forraje en los sistemas de alimentación de rumiantes en el trópico seco del sur de México. Sin embargo, aún se desconocen los factores que controlan su sobrevivencia en climas secos, con heladas, elevadas altitudes, entre los $2300-2500 \mathrm{~m}$ y suelos someros. En este estudio el modelo de nichos ecológicos mostró que para la microcuenca Tula su posible distribución es baja, pero en campo mostró alta capacidad de sobrevivencia. 


\section{Conclusiones}

El modelaje de nichos ecológicos con datos reducidos de presencia (menos de 20) demostró ser una herramienta confiable para determinar áreas con aptitud para especies arbustivas con fines de rehabilitación del suelo. La especie arbustiva evaluada $L$. collinsii depende principalmente de la estacionalidad de temperatura (BIO 4) y la evapotranspiración real anual de los meses húmedos (ETRAH), para su distribución potencial, la cual fue escasa, con apenas $8 \%$ de la superficie total de la microcuenca Tula.

Sin embargo, registró una alta sobrevivencia en la parcela experimental, lo que hace posible su introducción como una opción para rehabilitación de suelos degradados, pero hace falta realizar más estudios y determinar si la sobrevivencia en la etapa de establecimiento es un factor que debe considerarse para mantener un alto porcentaje de sobrevivencia después de un año del trasplante.

\section{Literatura citada}

Broennimann, O.; Treier, U. A.; Müller-Schärer, H.; Thuiller, W.; Peterson, A. T. and Guisan, A. 2007. Evidence of climatic niche shift during biological invasion. Ecology Letters. 8(10):701-709. https://doi.org/10.1111/j.1461-0248.2007.01060.x

Contreras-Medina, R.; Luna-Vega, I. y Ríos-Muñoz, C. A. 2010. Distribución de Taxus globosa (Taxaceae) en México: Modelos ecológicos de nicho, efectos del cambio del uso de suelo y conservación. Rev. Chilena de Historia Natural. 83(3):421-433. https://doi.org/10.4067/ S0716-078X2010000300009.

Crespo, G. 2008. Importancia de los sistemas silvopastoriles para mantener y restaurar la fertilidad del suelo en las regiones tropicales. Rev. Cubana Cienc. Agríc. 4(42):329-335.

Cruz-Cárdenas, G.; López-Mata, L.; Villaseñor, J. L. y Ortiz, E. 2014. Potential species distribution modeling and the use of principal component analysis as predictor variables. Rev. Mex. Biod. 85(1)189-199. https://doi.org/10.7550/rmb.36723.

Didan, K. 2015. MOD13A2 MODIS/Terra Vegetation Indices 16-Day L3 Global 1km SIN Grid V006 [Data set]. NASA EOSDIS Land Processes DAAC. https://doi.org/10.5067/ MODIS/MOD13A2.006.

Elith, J. H.; Graham, C. H.; Anderson, R. P.; Dudík, M.; Ferrier, S.; Guisan, A. and Zimmermann, N. E. 2006. Novel methods improve prediction of species' distributions from occurrence data. Ecography. 29(2):129-151. https://doi.org/10.1111/j.2006.0906-7590.04596.x.

Elith, J.; Kearney, M. and Phillips, S. 2010. The art of modelling range-shifting species. Methods in Ecology and Evolution. 1(4): 330-342. https://doi.org/10.1111/j.2041-210X.2010.00036.x.

Flores-Maldonado, J. J. 2014. Análisis de los patrones de diversidad de leguminosas arbustivas del río Sabinas, Coahuila, México; bajo el enfoque del modelado de nichos ecológicos. Tesis de Doctor en Ciencias con Acentuación en Manejo de Vida Silvestre y Desarrollo Sustentable. Facultad de Ciencias Biológicas, Universidad Autónoma de Nuevo León. San Nicolás de los Garza, Nuevo León, México. 122 p.

Guisan, A. and Zimmermann, N. E. 2000. Predictive habitat distribution models in ecology. Ecological Modeling. 135(2-3):147-186. https://doi.org/10.1016/S0304-3800(00)00354-9.

Hernández, C. A.; Alfonso, A. y Duquesne, P. 1987. Producción de carne basada en pastos naturales mejorados con leguminosas arbustivas y herbáceas II. Ceba final. Pastos y Forrajes. 1(10):246-252. 
Hijmans, R. J.; Cameron, S. E.; Parra, J. L.; Jones, P. G. and Jarvis, A. 2005. Very high resolution interpolated climate surfaces for global land areas. Inter. J. Climatol. 25(15):1965-1978. https://doi.org/10.1002/joc.1276.

Hutchinson, G. E. 1957. Concluding remarks. Cold spring harbor symposia on quantitative biology. 22(2):415-457.

INEGI. 2013a. Instituto Nacional de Estadística y Geografía. Censo de población y vivienda 2010. https://www.inegi.org.mx/programas/ccpv/2010/.

INEGI. 2013b. Instituto Nacional de Estadística y Geografía. Continúo de datos de perfiles de suelos. Escala 1:250 000. Serie II (Continuo Nacional). https://www.inegi.org.mx/temas/ edafologia/default.html\#Descargas.

INEGI. 2013c. Instituto Nacional de Estadística y Geografía. Continúo de elevaciones mexicano (CEM). https://www.inegi.org.mx/app/geo2/elevacionesmex/.

INEGI. 2017a. Instituto Nacional de Estadística y Geografía. Anuario estadístico y geográfico de Hidalgo 2017. México: Instituto Nacional de Estadística y Geografía. https://www.datatur.sectur.gob.mx/ITxEF_Docs/HGO_ANUARIO_PDF.pdf.

INEGI. 2017b. Instituto Nacional de Estadística y Geografía. Anuario estadístico y geográfico de México 2017. México: Instituto Nacional de Estadística y Geografía. https://www.inegi.org.mx/app/biblioteca/ficha.html?upc=702825097912.

INEGI. 2017c. Instituto Nacional de Estadística y Geografía. Anuario estadístico y geográfico de Tlaxcala 2017. México: Instituto Nacional de Estadística y Geografía. https://www.datatur.sectur.gob.mx/ITxEF_Docs/TLAX_ANUARIO_PDF.pdf.

INEGI. 2017d. Instituto Nacional de Estadística y Geografía. Conjunto de datos vectoriales de uso de suelo y vegetación. Escala 1:250000. http://www.conabio.gob.mx/ informacion/gis/.

López, G. M. A. 2007. Descripción y caracterización de nichos ecológicos: una visión más cuantitativa del espacio ambiental. Tesis de Maestría en Ciencias en Probabilidad y Estadística. Centro de Investigación en Matemáticas, A. C. Guanajuato, México. 93 p. https://cimat.repositorioinstitucional.mx/jspui/bitstream/1008/87/2/TE 244.pdf.

Martínez-Hernández, P. A.; Cortez-Díaz, E.; Purroy-Vásquez, R.; Palma-García, J. M.; Del PozoRodríguez, P. P. y Vite-Cristóbal, C. 2019. Leucaena leucocephala (LAM.) de Wit especie clave para una producción bovina sostenible en el trópico. Trop. Subtrop. Agroecosys. 22(1):331-357.

Martínez-Méndez, N.; Aguirre-Planter, E.; Eguiarte, L. E. y Jaramillo-Correa, J. P. 2016. Modelado de nicho ecológico de las especies del género Abies (Pinaceae) en México: Algunas implicaciones taxonómicas y para la conservación. Bot. Sci. 94(1):5-24. https://doi.org/10.17129/botsci.508.

Mullen, B. F.; Gabunada, F.; Shelton, H. M. and Stür, W. W. 2003. Agronomic evaluation of Leucaena. Part 2. Productivity of the genus for forage production in subtropical Australia and humid-tropical Philippines Agroforestry Systems. 58(2):93-107.

Osuna-Ceja, E. S.; Figueroa-Sandoval, B.; Martínez-Gamiño, M. A. y Pimentel-López, J. 2019. Un sistema agroforestal de secano para el altiplano semiárido de México. Rev. Mex. Cienc. Agríc. 22(especial):91-105.

Palma-Ordaz, S. y Delgadillo-Rodríguez, J. 2014. Distribución potencial de ocho especies exóticas de carácter invasor en el estado de Baja California, México. Bot. Sci. 92(4):587-597.

Pearson, R. G.; Raxworthy, C. J.; Nakamura, M. and Peterson, A. T. 2007. Predicting species distribution from small numbers of occurrence records: a test case using cryptic geckos in Madagascar. J. Biogeography. 34(1):102-117. https://doi.org/10.1111/j.1365-2699.2006. 01594.x. 
Peralta, J.; Zepeda, N. A. y Imbert, J. B. 2013. ¿Puede haber carrascales en el sur de Navarra? Una aproximación mediante la modelización de distribución de especies. Ecosistemas. 22(3):58-65. https://doi.org/10.7818/ECOS.2013.22-3.09.

Peterson, A. T.; Papeş, M. and Soberón, J. 2008. Rethinking receiver operating characteristic analysis applications in ecological niche modeling. Ecol. Model. 213(1):63-72. https://doi.org/10.1016/j.ecolmodel.2007.11.008.

Phillips, S. J. and Dudík, M. 2008. Modeling of species distributions with Maxent: new extensions and comprehensive evaluation. Ecography. 31(2):161-175. https://doi.org/10.1111/j.2007. 0906-7590.05203.x.

Phillips, S. J.; Anderson, R. P. and Schapire, R. E. 2006. Maximum entropy modeling of species geographic distributions. Ecol. Model. 190(3-4):231-259. https://doi.org/10.1016/j. ecolmodel.2005.03.026.

Pinto, R. R.; Medina, F. J.; Gómez, H.; Guevara, F. y Ley, A. 2013. Caracterización nutricional y forrajera de Leucaena collinsii a diferentes edades de corte en el trópico seco del sur de México. Revista de la Facultad de Agronomía-LUZ. 1(31):78-99.

Quesada-Quirós, M.; Acosta-Vargas, L. G.; Arias-Aguilar, D. y Rodríguez-González, A. 2016. Modelación de nichos ecológicos basado en tres escenarios de cambio climático para cinco especies de plantas en zonas altas de Costa Rica. Rev. Forestal Mesoam. Kurú. 34(14):0112. https://doi.org/10.18845/rfmk.v14i34.2991.

R Development Core Team. 2012. R: a language and environment for statistical computing. R Foundation for Statistical Computing, Vienna, Austria. 213 p.

Ramírez, D. M. 2011. Metodología para realizar y presentar los informes de sobrevivencia inicial (ISI) de las plantaciones forestales comerciales (aspectos técnicos). Comisipón Nacional Forestal, México. http://www.conafor.gob.mx:8080/documentos/ver.aspx?grupo=6\& articulo $=1564$.

Ramos-Font, M. E.; González, R. J. L. y Robles, C. A. B. 2015. Dispersión endozoócora de leguminosas silvestres: desde la recuperación hasta el establecimiento en campo. Ecosistemas. 24(3):14-21.

Reynoso, S. R.; Pérez, H. M. J.; López, B. W.; Hernández, R. J.; Muñoz, F. H. J.; Cob, U. J. V. y Reynoso, S. M. D. 2018. El nicho ecológico como herramienta para predecir áreas potenciales de dos especies de pino. Rev. Mex. Cienc. Forest. 48(9):47-68. https://doi.org/10.29298/rmcf.v8i48.114.

Sánchez, M. M. y Carvacho, B. L. 2011. Comparación de ecuaciones empíricas para el cálculo de la evapotranspiración de referencia en la Región del Libertador General Bernardo O’Higgins, Chile. Rev. Geogr. Norte Grande. 50(1):171-186.

Soberón, J. and Nakamura, M. 2009. Niches and distributional areas: concepts, methods, and assumptions. Proceedings of the National Academy of Sciences. 106(2):19644-19650. https://doi.org/10.1073/pnas.0901637106.

Soberón, J. and Peterson, A. T. 2005. Interpretation of model of fundamental ecological niches and species' distributional areas. Bio. Informatics. 2(1):1-10. https://doi.org/10.17161/bi.v2i0.4.

Turc, L. 1954. Le bilan d'eau des sols: Relations entre les precipitation, l'évaporation et l'écoulement. Annales Agronomiques. 1(5):491-595.

Valpassos, M. A. R.; Maltoni, K. L.; Cassiolato, A. M. R. and Nahas, E. 2007. Recovery of soil microbiological properties in a degraded area planted with Corymbia citriodora and Leucaena leucocephala. Sci. Agric. 64(1):68-72. https://doi.org/10.1590/S010390162007000100010 . 
Wencomo, H. B. y Ortiz, R. 2010. Comportamiento fenológico de 23 accesiones de Leucaena spp. Pastos y Forrajes. 4(33):1-12.

Zamora, E. M. C.; Buendía, E. J. C.; Martínez, H. P. A. y García, N. R. M. 2020. Diagnóstico del uso del suelo y vegetación en la microcuenca Tula, México. Rev. Mex. Cienc. Agríc. 11(1):57-68. https://doi.org/10.29312/remexca.v11i1.2213.

Zárate-Pedroche, S. 1998. La domesticación de Leucaena (Fabaceae, Mimosoideae) en México. Boletín de la Sociedad Botánica de México. 62(1):141-151. https://doi.org/10.17129/botsci.1557.

Zavala-Cruz, J.; Palma-López, D. J.; Fernández-Cabrera, C. R.; López-Castañeda, A. y ShirmaTórres, E. 2011. Degradación y conservación de suelos en la cuenca del Río Grijalva, Tabasco. Colegio de Postgraduados, Secretaría de Recursos Naturales y Protección Ambiental y PEMEX. Villahermosa, Tabasco, México. 89 p. 